RASĀYAN J. Chem.

Vol. 13 | No. 1 |245 - 248| January - March | 2020 ISSN: 0974-1496 | e-ISSN: 0976-0083 | CODEN: RJCABP

\title{
THEORETICAL VALIDATION OF MEDICINAL PROPERTIES OF Curcuma longa Linn
}

\author{
Sampat Suryawanshi and Pramod Kulkarni* \\ Department of Chemistry, Hutatma Rajguru Mahavidyalaya, Rajgurunagar, \\ Pune-410505, (Maharashtra) India \\ *E-mail: pramodskulkarni3@gmail.com
}

\begin{abstract}
Molecular property and bio-activity scores of seven curcuminoids derivative of Curcuma longa Linn present in Curcuma longa Linn were predicted using molinspiration software. For all the essential oil compounds, miLog $\mathrm{P}$ values were observed to be 5 this shows these compounds were not easily permeable across the cell membrane. TPSA in the assortment of $0.00-66.76$ (well below $160 \AA 2$ ) and $n$ violations $=1$ or 0 , molecular mass $<5$ [10], N0 of hydrogen bond donors $\leq 5$ (the sum of OHs and NHs), No of hydrogen bond acceptor $\leq 10$ (The sum of Os and Ns) were pragmatic for these compounds. This designates that these compounds were found to obey Lipinski's rule and can simply bind to the receptor and further used for the calculation of bioactivity score. The bioactivity score calculated by the activity score of GPCR ligand, ion channel modulator, nuclear receptor ligand, kinase inhibitor, protease inhibitor and enzyme inhibitor.
\end{abstract}

Keywords: Molinspiration, Curcuma longa Linn, Bioactivity Score, Lipinski’s Rule.

C RASĀYAN. All rights reserved

\section{INTRODUCTION}

Turmeric (Curcuma long L.) is a unique herb coalescing properties of ambrosial, pigment, ornamental and used as a remedy in a number of diseases. It is known as spice, herbal medicines, dyeing agents and cosmetics since the Vedic age. ${ }^{1}$ The importance of turmeric in wellbeing and nutrition has been significantly accepted since the discovery of the pharmaceutical properties of naturally occurring phenolic compounds in it. The dried rhizome of turmeric is an abundant source of beneficial phenolic compounds known as the curcuminoids. ${ }^{2}$

Curcuma longa Linn. is herbaceous perennial, and it placed in the ginger family (Zingiberaceae). It is innate to India and nowadays it is widely cultivated in the tropical and subtropical regions of south and Southeast Asia and some areas of Africa with a warm and wet tropical climate. Curcuma may invent from its Arabic name (kurkum) or Hebrew name (karkom) which means yellow. The term longa originates from the lengthened shape of its rhizome. The rhizome with a characteristic deep orange-yellow color, known as turmeric, is a very vital constituent in curry and extensively used as a coloring and flavoring agent. Turmeric is recognized by a variety of synonyms at universal, and numerous of these synonyms are allied to its key characteristic, orange-yellow color. Ranjani, Pita, and Varavarnini in India indicate the bright yellow color of the rhizome and Jiang-Huang in Chinese means yellow ginsger. ${ }^{3}$ Extensive study over the last five decades has observed that curcumin decreases blood cholesterol, stops LDL oxidation, inhibits platelet aggregation, quashes thrombosis, myocardial infarction, and quashes signs related with type II diabetes, rheumatoid arthritis, multiple sclerosis and Alzheimer's disease. ${ }^{4}$

Curcumioids are the core constituents of turmeric. The orange-yellow color of turmeric is mainly due to the highly unsaturated conjugated chemical structures of the curcumioids. The study on the isolation of the coloring principle of turmeric, curcumin, dated back to the $19^{\text {th }}$ century. Curcuminoids mentioned to a type of component in turmeric, which is chemically associated with its principal element curcumin. All curcuminoids have the same typical skeleton of two aromatic rings joined by an aliphatic chain, frequently a heptane chain. A few curcumioids have a 1,3-diketone group, which has a minimum two

Rasayan J. Chem., 13(1), 245-248(2020)

http://dx.doi.org/10.31788/RJC.2020.1315581

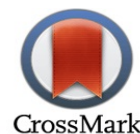


RASĀYAN J. Chem.

Vol. 13 | No. 1 | 245 - 248| January - March | 2020

Keto-Enol tautomers. The Enol form of curcumin preponderated in its Keto-Enol tautomeric mixtures in the solid phase and in solution. The Enol forms of the major curcuminoids have a huge conjugating system and have a UV-Vis absorption at $410-430 \mathrm{~nm}$, which gave its orange-yellow color. ${ }^{5}$

To date, 50 curcuminoids containing 3 characteristic sub types have been known: linear- curcuminoids, cyclic-curcuminoids and curcuminoids conjugated with monoterpenes or sesquiterpenes. Linear curcuminoids are the most frequently curcuminoids occurred in turmeric. The primary curcuminoids in turmeric are three linear curcuminoids including curcumin, demethoxycurcumin, and bisdemethoxycurcumin. Two cyclic diarylheptanoids bearing a furan ring have also been isolated from turmeric. Recently, a couple of uncommon curcuminoids with the structures conjugating curcuminoids, with monoterpenes or sesquiterpenes through $\mathrm{C}-\mathrm{C}$ or $\mathrm{C}-\mathrm{O}-\mathrm{C}$ bond were isolated from turmeric comprising 12 derivatives from the hybridization of curcuminoids and bisabolanes connected by $\mathrm{C}-\mathrm{O}$ bond, 15 conjugated curcuminoids with sesquiterpenes through $\mathrm{C}-\mathrm{C}$ bond, and 2 derivatives of bisdemethoxycurcumin joining with a menthane monoterpenoid. ${ }^{5}$

\section{Material and Methods}

\section{EXPERIMENTAL}

Structures of all the seven compounds reported from Curcuma longa Linn were captured from the literature. Their structures were drawn using online molinspiration software (www.molinspiration.com) ${ }^{6}$ for calculation of molecular properties ( Log P, Total polar surface area, number of hydrogen bond donors and acceptors, molecular weight, number of atoms, number of rotatable bonds etc.) and estimation of the bioactivity score for drug targets (GPCR ligands, kinase inhibitors, ion channel modulators, enzymes and nuclear receptors). The bioactivity score and drug-likeness properties of all the seven compounds were correlated.

\section{Prediction of Bio-activity}

1. Molecular properties of seven curcuminoids derivative of Curcuma longa Linn were calculated using molinspiration. The values of molecular properties were given in Table 1.

2. Bio-activity scores of seven curcuminoids derivative of Curcuma longa Linn calculated for GPCR ligand, ion channel modulator, nuclear receptor ligand, kinase inhibitor, protease inhibitor and enzyme inhibitors. The values of bio-activity scores were given in Table 2.

\section{Lipinski's Rule}

Lipinski's rule of five usually recognized as the Pfizer's rule of five or solely the Rule of five is a directive of thumb to the evaluation of drug-likeness or to classify a chemical compound with a positive pharmacological or biological activity has properties that would make it a probable orally active drug in man. The principle was intended by Christopher A. Lipinski in 1997. The rule states, molecular properties essential for a drug's pharmacokinetics in the human body, comprising their absorption, distribution, metabolism and elimination (ADME) Components of the Lipinski's rule. ${ }^{7,8}$

\section{Lipinski's the Rule States}

$>$ Not more than 5 hydrogen bond donors (nitrogen or oxygen atoms with one or more hydrogen atoms).

$>$ Not more than 10 hydrogen bond acceptors (nitrogen or oxygen atoms).

$>$ A molecular weight less than 500 .

$>$ An octanol-water partition coefficient $\log \mathrm{P}$ not greater than 5 .

$>$ No more than one number of violation

\section{Molinspiration}

Molinspiration, web-based software was applied to get parameters such as MiLogP, TPSA, drug-likeness. MiLogP, is measured by the procedure established by Molinspiration as a sum of fragment-based contributions and correction factors. MiLog P parameter is used to investigate good permeability across the cell membrane. TPSA is associated with the hydrogen bonding potential of the compound. The 
RASĀYAN J. Chem.

Vol. 13 | No. 1 |245 - 248| January - March | 2020

computation of volume elaborated at Molinspiration is established for group contributors. A number of rotatable bond measure molecular flexibility. It is a very good descriptor of the absorption and bioavailability of drugs. Through drug-likeness data's of a unit, it can be checked molecular properties and structure attributes with respect to recognized drugs.

\section{Bioactivity Score}

The bioactivity score of the drug can be established by computing the activity score of GPCR ligand, ion channel modulator, nuclear receptor ligand, kinase inhibitor, protease inhibitor, enzyme inhibitor. These parameters were investigated with the help of the software Molinspiration drug-likeness score online (www.molinspiration.com). A computed drug-likeness score of each compound and related to the specific bodily process of each compound. For organic molecules, the possibility is if the bioactivity score is $(>0)$, then it is active, if $(-5.0-0.0)$ then moderately active, if $(<-5.0)$ then inactive.
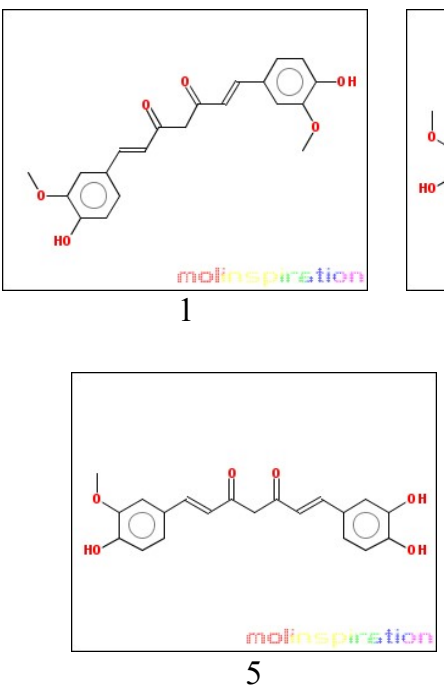

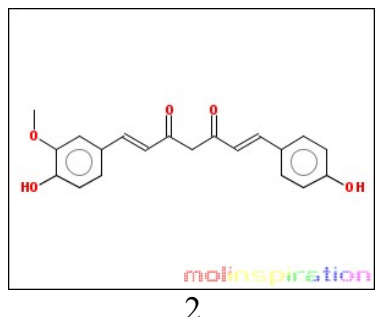

2

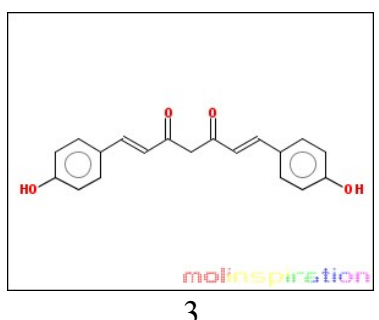

3

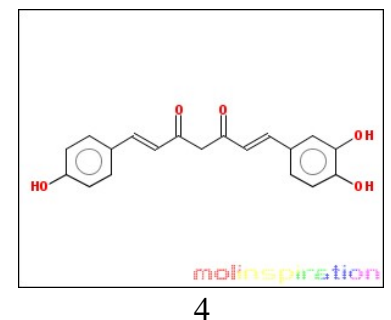

Fig.-1: Structure of Seven Curcuminoids Derivative of Curcuma longa Linn

Table-1: Calculation of Molecular Properties

\begin{tabular}{c|c|c|c|c|c|c|c|c|c|c}
\hline S. No. & Compound & miLogP & TPSA & natoms & MW & nON & noHNH & nviolations & nrobt & Volume \\
\hline 1 & 1 & 2.30 & 93.07 & 27 & 368.38 & 6 & 2 & 0 & 8 & 332.18 \\
\hline 2 & 2 & 2.48 & 83.83 & 25 & 338.36 & 5 & 2 & 0 & 7 & 306.64 \\
\hline 3 & 3 & 2.67 & 74.60 & 23 & 308.33 & 4 & 2 & 0 & 6 & 281.09 \\
\hline 4 & 4 & 2.18 & 74.60 & 24 & 324.33 & 5 & 3 & 0 & 6 & 289.11 \\
\hline 5 & 5 & 2.00 & 104.06 & 26 & 354.36 & 6 & 3 & 0 & 7 & 314.65 \\
\hline 6 & 6 & 1.69 & 115.05 & 25 & 340.33 & 6 & 4 & 0 & 6 & 297.13 \\
\hline 7 & 7 & 2.01 & 113.29 & 28 & 384.38 & 7 & 3 & 0 & 8 & 340.20 \\
\hline
\end{tabular}

Table-2: Bioactivity Score

\begin{tabular}{c|c|c|c|c|c|c|c}
\hline S. No. & Compound & GPCR Ligand & $\begin{array}{c}\text { Ion Channel } \\
\text { Modulator }\end{array}$ & $\begin{array}{c}\text { Kinase } \\
\text { Inhibitor }\end{array}$ & $\begin{array}{c}\text { Nuclear Receptor } \\
\text { Ligand }\end{array}$ & $\begin{array}{c}\text { Protease } \\
\text { Inhibitor }\end{array}$ & $\begin{array}{c}\text { Enzyme } \\
\text { Inhibitor }\end{array}$ \\
\hline 1 & 1 & -0.06 & -0.20 & -0.26 & 0.12 & -0.14 & 0.08 \\
\hline 2 & 2 & -0.04 & -0.20 & -0.26 & 0.18 & -0.14 & 0.10 \\
\hline 3 & 3 & 0.00 & -0.14 & -0.26 & 0.25 & -0.08 & 0.15 \\
\hline 4 & 4 & 0.01 & -0.13 & -0.24 & 0.26 & -0.08 & 0.15 \\
\hline 5 & 5 & -0.06 & -0.21 & -0.27 & 0.12 & -0.14 & 0.08 \\
\hline 6 & 6 & -0.01 & -0.15 & -0.26 & 0.20 & -0.07 & 0.13 \\
\hline 7 & 7 & -0.07 & -0.20 & -0.24 & 0.10 & -0.13 & 0.10 \\
\hline
\end{tabular}


RASĀYAN J. Chem.

Vol. 13 | No. 1 | 245 - 248| January - March | 2020

\section{Molecular Property of The Curcuminoids}

\section{RESULTS AND DISCUSSION}

The seven curcuminoids derivative of Curcuma longa Linn complies the Lipinski's rule of five and exhibited good drug-likeness scores. MiLog P values of these curcuminoids compounds were observed to be $<5$ (1.69-2.67 for compounds 1 to 7$)$ showed their good permeability across the cell membrane. These compounds were observed to have TPSA will be below $160 \AA 2(100.13)$, molecular weight $<500$, No. of hydrogen bond donors $\leq 5$, No. of hydrogen acceptor $\leq 10$, n-violations 0 , number of rotatable flexible bonds $>5$.

\section{Bioactivity Scores of The Components of Curcuma Longa Linn}

The studies of bioactivity scores of these compounds have the following observations:

1. GPCR Ligand: Among the seven compounds, compounds 1, 2, 5, 6, and 7were observed to be moderately active $(\leq 0)$. The compounds 3 and 4 were found highly active

2. Ion channel modulator: These compounds were found to be moderately $(\leq 0)$ concerned with ion channel modulator $(\leq 0)$.

3. Kinase inhibitor: All seven compounds were observed to be inactive $(\leq 0)$ with respect to Kinase inhibitor.

4. Nuclear receptor ligand: All the seven compounds, were observed to be highly active $(\geq 0)$ towards Nuclear receptor ligand.

5. Protease inhibitor: These compounds, were to be moderately active $(\leq 0)$ towards Protease inhibitor.

6. Enzyme inhibitor: All the seven compounds, highly active $(\geq 0)$ towards Enzyme inhibitor.

\section{CONCLUSION}

In conclusion, seven curcuminoids derivative of Curcuma longa Linn observed highly active to moderate bioactivity score. All compounds confirmed Lipinski's rule for Drug Likeness activity of the molecules.

\section{ACKNOWLEDGMENT}

Authors thanks to DST FIST (Project File No. SR/FST/College-296/2015) for providing the Grant for Computer Networking Facility

\section{REFERENCES}

1. N. D. Salvi, L. George, S. Eapen, Plant Cell Tissue Organ Culture, 62, 235(2000), DOI:10.1023/A:1006459822879

2. K. R. Srinivasan, Journal Pharmacy and Pharmacology, 5, 448(1953), DOI:10.1111/j.20427158.1953.tb14007.x

3. F. -C. Meng, Y.-Q. Zhou, D. Ren, R. Wang, C. Wang, L. -G. Lin, X. -Q. Zhang, W.-C. Ye, Q. -W. Zhang, Handbook of Food Bioengineering, pp. 299-350(2018), DOI:10.1016/B978-0-12-8115183.00010-7.

4. A. Akbar, A. Kuanar, R. Joshi, I. Sanddep, S. Mohanty, P. Naik, A. Mishra, S. Nayak, Frontiers Plant Science, 7, 1507(2016), DOI:10.3389/fpls.2016.01507.

5. T. T. Dao, P. H. Nguyen, H. K. Won, E. H. Kim, J. Park, B. Y. Won, W. K. Oh, Food Chemistry, 134, 21(2012), DOI:10.1016/j.foodchem.2012.02.015.

6. http://www.molinspiration.com

7. C. A. Lipinski, F. Lombardo, B. W. Dominy, P. J. Feeney, Advanced Drug Delivery Reviews, 23, 3(1997), DOI:10.1016/S0169-409X(96)00423-1.

8. C. A. Lipinski, F. Lombardo, B. W. Dominy, P. J. Feeney, Advanced Drug Delivery Reviews, 46, 3(2001), DOI: 10.1016/S0169-409X(00)00129-0

[RJC-5581/2019] 